\title{
MSLQ: INSTRUMENT VALIDATION OF MOTIVATION AND LEARNING STRATEGIES FOR ACQUIRING COMPUTER SOFTWARE APPLICATION SKILLS
}

\author{
Sheila M. Smith, Ball State University, smariesmith@bsu.edu \\ Catherine Chen, Ball State University, cchen@bsu.edu
}

\begin{abstract}
Learning specialized computer skills necessitates using the computer as a problem-solving tool while displaying superior affective skills in an applied learning environment. Motivational Strategies for Learning Questionnaire (MSLQ) motivation scale assesses students' general motivation to learn, but does not capture students' motivation and use of effective learning strategies in an applied learning environment. To better understand the difference (or lack of difference) in students' motivation to engage in problem-based learning, a valid instrument is needed. Exploratory factor analyses and confirmatory factor analyses showed that the survey items did not confirm to the original Motivation and Learning Strategies scales. The refined Motivation section consisted of 24 items in three scales: Expectancy, Task Value, and Test Anxiety. The refined Learning Strategies section consisted of 25 items in four scales: Effort Regulation, Organization, Critical Thinking, and General Strategies for Learning.
\end{abstract}

Keywords: MSLQ, Computer-based Learning, Motivation, Learning Strategies, Validity Testing

\section{INTRODUCTION}

Current learning theories view students as active participants in their own learning process, and they are regarded as self-regulated learners. Based on this and social-cognitive theories, the Motivated Strategy for Learning Questionnaire (MSLQ) was developed to assess college students' motivation and their use of learning strategies, as well as to investigate the relationships between motivation, use of learning strategies, and academic performance [7, 8, 9]. Although MSLQ has been used in hundreds of studies, researchers have reported two concerns. First, social-cognitive theories suggest that students' motivation and their use of learning strategies are context-specific; however, the MSLQ was designed to be used in any subjects and for all types of learning activity. Second, psychometric problems were identified. Several studies confirmed that some sub-scales in the MSLQ lack discriminant validity and that the instrument had poor latent factor structure $[1,5]$.

Learning to use computer software applications is very different from conceptual textbook learning. Learning to use software requires students to 'use' various software features to solve a problem or to produce a product (such as an architectural design). In contrast, conceptual textbook learning usually requires students to attend lectures and take notes while learning concepts and theories. To respond to the need for context-specificity, a study has been conducted to modify the motivation and learning strategies items specifically for learning computer software applications and to test the confirmatory and discriminant validity of this adapted MSLQ. Therefore, the primary purpose of this study was to construct MSLQ context-specific motivation and learning items and to test its latent factor structures, validity, and reliability.

\section{REVIEW OF LITERATURE}

Recent research had revealed concerns about the latent factor structure of the MSLQ. According to Hilpert, Stemien, van der Hoeven Kraft, and Husman [5], the confirmatory factor analysis (CFA) did not produce a good fit in its first development in 1990. An attempt was made to improve the fit in 1993 without success. To determine if there is a reasonable model fit, using the original MSLQ, Hilpert et al., [5], examined the latent factor structure of the entire scale using data from a single administration of the instrument. Three general analyses were conducted. First, descriptive statistics, internal reliability estimates, and correlations for all MSLQ sub-scales were computed, data screening, and an inspection of the variance components for the individual sub-scales to determine if betweenuniversity variations required multilevel modeling. Second, based upon an orthogonal model a baseline was established for the latent factor structure of the five MSLQ scales - Expectancy, Value, Affect, Cognitive Strategy 
Use, and Resource Management. Finally, the final model was formulated which provided evidence for a three-factor model with six sub-scales. By eliminating sub-scales that showed inadequate performance, the latent structure consisted of expectancy (self-efficacy and control of learning beliefs), value (intrinsic motivation and task value), and self-regulation (metacognitive self-regulation and effort regulation).

Dunn, Lo, Mulvenon, and Sutcliffe [3] evaluated two of the Learning Strategies sub-scales - the Metacognitive SelfRegulation (MSR) sub-scale and the Effort Regulation (ER) sub-scale. The MSR scale with 12 items assessed one's aptitude for three general regulatory processes (i.e., planning, monitoring, and regulating) and the ER sub-scale with four items assesses learners' ability to manage resources [9]. Dunn et al. [3] sought to statistically and theoretically reexamine the MSR and the ER scales due to debate over construct uniqueness and respondent's inability to distinguish between the two sub-scales. In addition, the study examined how well individual items loaded onto the targeted latent constructs. Exploratory factor analysis was used to eliminate measurement items that did not load substantially on any factor. Two latent factors renamed as General Strategies for Learning (GSL) and Clarification Strategies for Learning (CSL) emerged. The GSL consisted of five items that included questions related to general self-regulation strategies. The second set of items was labeled as CSL and consisted of three items that included questions related to identifying and clarifying confusion and misunderstandings in the learning process. According to Dunn et al. [3], the GSL and CSL scales provide an up-to-date and relevant method to assess students' aptitude for the fundamental academic ability of self-regulation.

Cook, Thompson, and Thomas [1] conducted a validity study evaluating the relationships of MSLQ scores to other variables and their internal structure (reliability and factor analysis). Internal consistency was universally high for all MSLQ factors and sub-scales. Total MSLQ scores showed statistically significant positive associations with post-test knowledge scores. Total MSLQ scores showed moderately strong, statistically significant associations with effort, motivation and satisfaction. Self-efficacy and control of learning belief scores demonstrated the strongest domainspecific relationships with knowledge scores. Confirmatory factor analysis showed a borderline data-model fit. The follow-up exploratory factor analysis produced a five-factor model (self-efficacy, intrinsic interest, test anxiety, extrinsic goals, and attribution).

Rotgans and Schmidt [10] used the original MSLQ and a context-specific modified MSLQ to determine whether the MSLQ was dependent on the learning context (static) or generalizable across academic subject domains (stable). Both the original and modified versions of the MSLQ revealed that most latent means scores for both learning and motivation across different academic subject domains were not significantly different. Although the results were insignificant, the modified MSLQ did indicate slight differences between academic subject domains. Based upon the modified MSLQ motivation model, mean differences appeared in students' self-efficacy and task-value of math, science, and English. The study revealed that the motivation factor varies. Latent mean and predictive validity analysis indicated that self-efficacy and task-value beliefs were context-specific. In addition, mean differences were found in the learning strategy model for metacognitive self-regulation, effort regulation, and elaboration between math, science, and English. However, the study emphasized the point that self-regulated learning is not highly context-dependent and is not situation-specific to learning activities.

\section{RESEARCH METHODOLOGY}

This section describes the original scales in the MSLQ, the steps taken to revise the MSLQ items, the subjects participated in the study, the data collection procedures, and the statistical analyses used.

\section{The Original Scales}

The Motivation section of the original MSLQ consisted of 31 items in six scales: Intrinsic Goal Orientation, Extrinsic Goal Orientation, Task Value, Control of Learning Belief, Self-Efficacy for Learning and Performance, and Test Anxiety. The Learning Strategies section consisted of 50 items in nine scales: Rehearsal, Elaboration, Organization, Critical Thinking, Metacognitive Self-Regulation, Time and Study Environment, Effort Regulation, Peer Learning, and Help Seeking [8]. 


\section{The Revisions of the Questionnaire Items}

Two senior instructors, each with more than 20 years of teaching computer-related classes, revised the items, and made them more specifically focused on learning computer skills. The revised items were given to four students, two male and two female, majoring in Information Systems to solicit feedback on the wording and clarity of the revised items. Based on these students' feedback, minor changes were made. These students also recommended to move similar sounding items farther apart.

\section{Subjects}

Students enrolled in several computer-related classes in the Information Systems and Operations Management Department at a large Midwestern university were invited to participate in this study. Students enrolled in these classes were required to use two or three of the following tools: Word, PowerPoint, Excel, Access, Visio, Project, Visual Basic for Applications (VBA), Windows Server 2012 R2, and the Web/Internet. The revised instrument was created using Qualtrics, and responses were scored using a 6-point Likert type scale, from 1 (not at all like me) to 6 (just like $m e)$.

A total of 459 students participated in the Motivation survey. Of the 459 students, 302 were male $(65.8 \%)$ and 152 were female (33.1\%) students. In terms of age, the majority of students were in their early $20 \mathrm{~s}: 17 \%$ were 20 years old, $32.7 \%$ were 21 years old, and $20 \%$ were 22 years old. The majority of students identified themselves as White $(81.5 \%)$. Thirty-eight percent were in their senior year, $34.4 \%$ were in their junior year, $13.1 \%$ were in their sophomore, and $13.3 \%$ were in their freshman year, with $9 \%$ of students did not provide responses to the question. The majority of students (85\%) were enrolled in the College of Business, $9.4 \%$ were enrolled in Applied Science and Technology College, with a few enrolled in Fine Arts, Sciences and Humanities, or the Teacher's College.

A total of 203 students participated in the Learning Strategies survey. The demographics were similar to that of the students who participated in the Motivation survey. One hundred thirty-three students $(65.5 \%)$ were male and 65 (32\%) were female students. The majority of this group of students were also in their early $20 \mathrm{~s}: 12.8 \%$ were 20 years old, $35.5 \%$ were 21 years old, and $21.7 \%$ were 22 years old. Eighty-one percent of students identified themselves as White. Fifty-four percent were in their senior year, $31.5 \%$ were in their junior year, $3.4 \%$ were in their sophomore year, and $9.9 \%$ were in their freshman year. One hundred and seventy-eight students $(87.8 \%)$ were enrolled in the College of Business, twenty-two students (10.8\%) were majored in Applied Science and Technology, and a few students were enrolled in Fine Arts, Sciences and Humanities, or the Teacher's College.

\section{Data Collection Procedures}

Data were collected multiple times for this study. Data for the Motivation section were collected during the week prior to the last week of the spring and fall semesters in 2014. The data for the Learning Strategies were collected in the week prior to the last week of fall semester in 2014. Consent to collect data was obtained from each of the instructors who taught the course. On the prearranged day, an assistant used the last 15 minutes of the class to collect the data after the instructor who taught the class had left the classroom. Students were invited to complete the survey online using university computers so that their responses would not be identifiable. The online survey included an opening section detailing the purposes of the study, the voluntary nature of their participation, and the anonymity of their responses. In addition, the instruction stated that their action of participation or nonparticipation and their responses would not affect their course grade. Student selected a consent button before they were allowed to proceed to the revised motivation survey.

\section{Analysis Approach}

EFA using principal components factor extraction was used to determine if the items in the Motivation section and those in the Learning Strategies section correspond to the respective scales. To ensure that it was appropriate to perform factor analysis on the data, measure of sample adequacy (MSA) statistics were calculated prior to performing the factor analysis [4]. 
Validity refers to the degree of adequacy and appropriateness of the interpretations and actions based on the observed scores [4]. In this study, CFA was used to investigate the internal validity. A path model was used as a basis for testing convergent validity and discriminant validity. Both convergent and discriminant evidence are fundamental in testing validation [4]. Convergent validity measures constructs that theoretically should be related to each other are, in fact, observed to be related to each other. Discriminant validity, on the other hand, demonstrates measures that should not be related are in reality not related. CFA was used to test both validity in this study. Following the factor analyses, the internal consistency of reliability was computed using Cronbach's coefficient alphas [2].

\section{FINDINGS}

This section reports the findings of the MSA, EFA, and CFA. The data collected in the Motivation section and the data collected in the Learning Strategies section were analyzed separately, and the findings are reported in the following sections.

\section{Motivation Scales}

This section details the findings in the findings of the initial MSA, EFA, and CFA. Then, it describes the process used to refine the model and the findings of the final Motivation scales.

Exploratory Factor Analysis. The data were subjected to an MSA prior to the EFA. The overall KMO (MSA) statistic was .963, and all item MSAs exceeded the desired value of .7; therefore, the MSA criteria were satisfied. The EFA result showed that all but item 25 had a communality value of .312. Communality values were the variance explained for each item. A low communality suggests that the factor model was not working well for that indicator, and a communality of.5 was desired. Four factors were extracted which accounted for $69.67 \%$ of the variance. As shown in Table 1, the factor loadings did not correspond well to the original Motivation scales. With the exception of Test Anxiety. Although Factor 1 loaded well on the Extrinsic scale, all other scale items that loaded on this scale was problematic. Items in Intrinsic, Task Value, Control Beliefs, and Self-efficacy all loaded on multiple factors.

Table 1. Initial Motivation Scales Factor Loadings

\begin{tabular}{|l|c|c|c|c|l|l|l|l|l|}
\hline & \multicolumn{5}{|c|}{ Component } & \multicolumn{3}{c|}{ Component } \\
\cline { 2 - 6 } & $\mathbf{1}$ & $\mathbf{2}$ & $\mathbf{3}$ & $\mathbf{4}$ & & $\mathbf{1}$ & $\mathbf{2}$ & $\mathbf{3}$ & $\mathbf{4}$ \\
\hline M1_I & .563 & & & & M5_SE & .738 & & & \\
\hline M16_I & & & & .742 & M6_SE & .433 & & & .539 \\
\hline M22_I & .615 & & & & M12_SE & .909 & & & \\
\hline M24_I & & & .636 & & M15_SE & .499 & & & .484 \\
\hline M7_E & .836 & & & & M20_SE & .794 & & & \\
\hline M11_E & .848 & & & & M21_SE & .903 & & & \\
\hline M13_E & .916 & & & & M29_SE & .676 & & & \\
\hline M30_E & .645 & & & & M31_SE & .758 & & & \\
\hline M4_TV & .726 & & & & M3_TA & & .774 & & \\
\hline M10_TV & .756 & & & & M8_TA & .529 & .554 & & \\
\hline M17_TV & & & .930 & & M14_TA & .600 & .520 & & \\
\hline M23_TV & .672 & & & & M19_TA & & .848 & & \\
\hline M26_TV & & & .801 & & M28_TA & & .785 & & \\
\hline M27_TV & .419 & & .603 & & & & & & \\
\hline M2_CB & .926 & & & & & & & & \\
\hline M9_CB & .499 & & & & & & & & \\
\hline
\end{tabular}




\begin{tabular}{|l|c|c|c|c|c|c|c|c|c|}
\hline & \multicolumn{4}{|c|}{ Component } & \multicolumn{4}{|c|}{ Component } \\
\cline { 2 - 8 } & $\mathbf{1}$ & $\mathbf{2}$ & $\mathbf{3}$ & $\mathbf{4}$ & & $\mathbf{1}$ & $\mathbf{2}$ & $\mathbf{3}$ & $\mathbf{4}$ \\
\hline M18_CB & .869 & & & & & & & & \\
\hline M25_CB & & & .469 & & & & & & \\
\hline
\end{tabular}

Confirmatory Factor Analysis. A path model was created based on the six original scales in the Motivation section of the instrument. According to Hair, Black, Babin, Anderson, and Tatham [4], for a study with more than 250 subjects and more than 30 items, the characteristics of a good fit include a CFI of above .90, a GFI of above .90, and a RMSEA of below .7. The GFI for this model was .691, the CFI was .841, and the RMSEA was .104. Although the fit of the model falls short of satisfying fit criteria, convergent validity was conducted. Convergent validity shows items in the same motivation scale were correlated. There were two tests for convergent validity. The first was to test if the unstandardized regression weights (path coefficients) were statistically significant. The second was to test if the factor loadings (standardized regression weights) were .5 or higher. For the Motivation scales, the first test of convergent validity was satisfied with all path coefficients being statistically significant. The second test of convergent validity was mostly confirmed since all but three items (items 16,17, and 25) had satisfactory factor loadings of higher than .5 .

To test discriminant validity, the comparison of root average variance explained and inter-scale correlation was produced for the model. Scales exhibited discriminant validity when root average variances were larger than correlations in the same row and column in the table. As shown in Table 2, only Test Anxiety exhibited discriminant validity.

Table 2. Comparison of the Root Average Variance Explained and Inter-Scale Correlation for Motivation Scales

\begin{tabular}{|r|r|r|r|r|r|}
\hline \multicolumn{1}{|c|}{ Extrinsic } & Control Beliefs & \multicolumn{1}{|c|}{ Self-Efficacy } & Test Anxiety & \multicolumn{1}{c|}{ Intrinsic } & \multicolumn{1}{c|}{ Task Value } \\
\hline 0.837 & & & & & \\
\hline 0.872 & 0.749 & & & & \\
\hline 0.828 & 0.951 & 0.825 & & & \\
\hline 0.663 & 0.452 & 0.363 & 0.687 & & \\
\hline 0.806 & 0.905 & 0.891 & 0.508 & 0.654 & 0.809 \\
\hline 0.821 & 0.886 & 0.855 & 0.499 & 0.957 & \\
\hline
\end{tabular}

Refinement of the Motivation Scales. Several iterations of EFA and CFA were conducted to refine the Motivation scales. In general, items with an MSA of lower than .5 are removed. Cross loading occurs when a variable had two or more significant factor loadings in an EFA. With a sample size of 350 or greater, a loading of 30 was considered significant [4]. Items with significant cross loadings were deleted. The final Motivation scales consisted of 24 items and 3 scales. Table 3 shows the factor loading. Test Anxiety scale is the only scale that retained its original items, although it was reduced from five to three items. Of the 18 items, 9 was from the original Self-Efficacy scale and 2 were from the Control Beliefs scale. Hilpert et al, had similar findings [5] and named the combination of Self-Efficacy and Control Beliefs as Expectancy. It was determined to re-label Factor 1 as Expectancy.

Table 3. Final Motivation Scales Factor Loadings

\begin{tabular}{|l|c|c|c|c|c|c|c|}
\hline & \multicolumn{3}{|c|}{ Component } & & \multicolumn{3}{c|}{ Component } \\
\cline { 2 - 4 } \cline { 7 - 8 } & $\mathbf{1}$ & $\mathbf{2}$ & $\mathbf{3}$ & & $\mathbf{1}$ & $\mathbf{2}$ & $\mathbf{3}$ \\
\hline M2_CB & .945 & & & M19_TA & & .872 & \\
\hline M12_SE & .942 & & & M28_TA & & .831 & \\
\hline M13_E & .927 & & & M3_TA & & .704 & \\
\hline M21_SE & .910 & & & M17_TV & & & .872 \\
\hline M18_CB & .904 & & & M26_TV & & & .751 \\
\hline M11_E & .853 & & & M24_I & & & .706 \\
\hline M7_E & .853 & & & & & & \\
\hline M20_SE & .795 & & & & & & \\
\hline
\end{tabular}




\begin{tabular}{|l|l|l|l|l|l|l|l|}
\hline M10_TV & .794 & & & & & & \\
\hline M31_SE & .786 & & & & & & \\
\hline M4_TV & .761 & & & & & & \\
\hline M29_SE & .740 & & & & & & \\
\hline M5_SE & .733 & & & & & & \\
\hline M23_TV & .726 & & & & & & \\
\hline M30_E & .679 & & & & & & \\
\hline M22_I & .650 & & & & & & \\
\hline M1_I & .611 & & & & & & \\
\hline M6_SE & .498 & & & & & & \\
\hline
\end{tabular}

Based on the factors identified in this EFA, a path model was created for the CFA. The model fit characteristics included a CFI of .917, a GFI of .819, and a RMSEA of .09. The CFI was larger than the desired .90 and both GFI and RMSEA were close to the desired $>.90$ and $<.07$, respectively. Convergent validity was exhibited: all path coefficients were statistically significant and all factor loadings were higher than .5. In addition, all three scales exhibited discriminant validity. The root average variance explained was larger than correlations in the same row and column in the table. Table 4 shows the comparison of root average variance and inter-scale correlations for the final Motivation Scales.

Table 4. Comparison of Root Average Variance and Inter-Scale Correlation for the final Motivation Scales

\begin{tabular}{|c|c|c|}
\hline $\begin{array}{c}\text { Test } \\
\text { Anxiety }\end{array}$ & Expectancy & Task Value \\
\hline 0.766 & & \\
\hline 0.158 & 0.806 & \\
\hline 0.117 & 0.633 & 0.788 \\
\hline
\end{tabular}

Reliability. Cronbach's Alpha was calculated for each of the scales. All Cronbach's Alpha met the desired .7: SelfEfficacy (.971), Test Anxiety (.781), and Task Value (.817). No deletion of any item would raise the scale Alpha.

\section{Learning Strategies Scales}

The same statistic procedures were used to analyze the data collected for the Learning Strategies scales and to refine the model.

Exploratory Factor Analysis. The data for Learning Strategies were collected in a different study with a total of 203 students participated in the study. The overall KMO (MSA) statistic for this study was .872, but three item MSAs did not reach the desired value of .7: item 2(.695), item 4 (.691), and item 9 (.601). Since these were fairly close to the desired value of .7, an EFA was conducted. Unfortunately, rotation failed to converge. Since there were nine scales in the Learning Strategies section, a second EFA was conducted, forcing the number of factors to nine. The EFA result showed that the communality values of all but three items 25 did not exceed the desired value of .5: item 5(.457), item 23 (.447), and item 27 (.413). The model accounted for $60.267 \%$ of the variance. However, the factor loadings did not correspond to the pre-specified Learning Strategies scales, as shown in Table 5.

Table 5. Initial Learning Strategies Factor Loadings

\begin{tabular}{|l|c|c|c|c|c|c|c|c|c|}
\hline & \multicolumn{7}{|c|}{ Component } \\
\cline { 2 - 10 } & $\mathbf{1}$ & $\mathbf{2}$ & $\mathbf{3}$ & $\mathbf{4}$ & $\mathbf{5}$ & $\mathbf{6}$ & $\mathbf{7}$ & $\mathbf{8}$ & $\mathbf{9}$ \\
\hline LS15_R & & .505 & & .495 & & & & & \\
\hline LS27_R & & & & & & & & & \\
\hline LS28_R & .729 & & & & & & & & \\
\hline LS41_R & & .683 & & & & & & & \\
\hline LS22_E & .664 & & & & & & & & \\
\hline LS31_E & .537 & & & & .404 & & & & \\
\hline
\end{tabular}




\begin{tabular}{|l|l|l|l|l|l|l|l|l|l|}
\hline LS33_E & .682 & & & & & & & & \\
\hline LS36_E & & .688 & & & & & & & \\
\hline LS38_E & .772 & & & & & & & & \\
\hline LS50_E & .744 & & & & & & & & \\
\hline LS1_O & & .555 & & & & & & & \\
\hline LS11_O & .550 & & & .463 & & & & & \\
\hline LS18_O & & .508 & & .423 & & & & & \\
\hline LS32_O & & .721 & & & & & & & \\
\hline LS7_CT & & & & & .653 & & & & \\
\hline LS16_CT & & & & .789 & & & & & \\
\hline LS20_CT & & & & .565 & & & & & \\
\hline LS35_CT & & & & .479 & .507 & & & & \\
\hline LS40_CT & .529 & & & & .496 & & & & \\
\hline
\end{tabular}

Table 5. Initial Learning Strategies Factor Loadings (cont.)

\begin{tabular}{|c|c|c|c|c|c|c|c|c|c|}
\hline & \multicolumn{9}{|c|}{ Component } \\
\hline & 1 & 2 & 3 & 4 & 5 & 6 & 7 & 8 & 9 \\
\hline LS2_SR & & & .643 & & & & & & \\
\hline LS5_SR & & & & & & & & & \\
\hline LS10_SR & .760 & & & & & & & & \\
\hline LS13_SR & .636 & & & & & & & & \\
\hline LS23_SR & .493 & & & & & & & & \\
\hline LS24_SR & & & & .479 & .501 & & & & \\
\hline LS25_SR & .556 & .422 & & & & & & & \\
\hline LS26_SR & & & .615 & & & & & & \\
\hline LS30_SR & .421 & & -.444 & & .408 & & & & \\
\hline LS45_SR & .729 & & & & & & & & \\
\hline LS47_SR & & & & & & & .650 & & \\
\hline LS48_SR & .416 & & & & & & .477 & & \\
\hline LS4_TS & & & & & & .619 & & & \\
\hline LS12_TS & .522 & & & & & & & & \\
\hline LS21_TS & & & .574 & & & & .404 & & \\
\hline LS34_TS & .435 & & & & & & & & \\
\hline LS39_TS & .538 & .483 & & & & & & & \\
\hline LS42_TS & .797 & & & & & & & & \\
\hline LS46_TS & & & .669 & & & & & & \\
\hline LS49_TS & & & .612 & & & & & & \\
\hline LS6_ER & & & .668 & & & & & & \\
\hline LS17_ER & .695 & & & & & & & & \\
\hline LS29_ER & & & .651 & & & & & & \\
\hline LS43_ER & .812 & & & & & & & & \\
\hline LS3_PL & & & & & & & & .783 & \\
\hline LS14_PL & .516 & & & & & .491 & & & \\
\hline LS19_PL & & & & & & .574 & & & \\
\hline
\end{tabular}




\begin{tabular}{|l|l|l|l|l|l|l|l|l|l|}
\hline LS8_HS & & & & .514 & & & & & \\
\hline LS9_HS & & & & & & & & & -.748 \\
\hline LS37_HS & .613 & & & & & & & & \\
\hline LS44_HS & .530 & & & & & .458 & & & \\
\hline
\end{tabular}

Confirmatory Factor Analysis. A path model was created based on the nine original scales in the Learning Strategies section of the MSLQ. In a study with fewer than 250 subjects and more than 30 items, the characteristics of a good fit include a CFI of above .92, a GFI of above .92, and a RMSEA of below .08 [4]. The GFI for this model was .540, the CFI was .579, and the RMSEA was .091. Although the fit of the model falls short of satisfying fit criteria, convergent validity was conducted.

To achieve convergent validity, the path coefficients should be statistically significant and the factor loadings should be .5 or higher. In this initial model, the path coefficients for most items in the Self-Regulation and Time and Study Environment scales were not statistically significant. Therefore, the convergent validity tests failed.

To achieve discriminant validity, a root average variance explained need to be larger than correlations in the same row and column in the table. As shown in Table 6, none of the scales exhibited discriminant validity. 
Table 6. Comparison of the Initial Root Average Variance and Inter-Scale Correlation-Learning Strategies Scales

\begin{tabular}{|c|c|c|c|c|c|c|c|c|}
\hline Elaboration & Rehearsal & $\begin{array}{c}\text { Effort } \\
\text { Regulation }\end{array}$ & $\begin{array}{c}\text { Peer } \\
\text { Learning }\end{array}$ & $\begin{array}{c}\text { Self- } \\
\text { Regulation }\end{array}$ & $\begin{array}{c}\text { Time and Study } \\
\text { Environment }\end{array}$ & $\begin{array}{c}\text { Help } \\
\text { Seeking }\end{array}$ & $\begin{array}{c}\text { Critical } \\
\text { Thinking }\end{array}$ & Organization \\
\hline 0.654 & & & & & & & & \\
\hline 0.790 & 0.458 & & & & & & & \\
\hline 0.862 & 0.629 & 0.587 & & & & & & \\
\hline 0.618 & 0.570 & 0.439 & 0.550 & & & & & \\
\hline 1.006 & 0.863 & 0.854 & 0.671 & 0.496 & & & & \\
\hline 0.961 & 0.824 & 1.036 & 0.659 & 1.018 & 0.428 & & & \\
\hline 0.874 & 0.606 & 0.725 & 0.912 & 0.754 & 0.830 & 0.530 & & \\
\hline 0.642 & 1.025 & 0.427 & 0.562 & 0.709 & 0.489 & 0.490 & 0.596 & \\
\hline 0.599 & 1.125 & 0.524 & 0.608 & 0.762 & 0.690 & 0.442 & 0.714 & 0.578 \\
\hline
\end{tabular}

Refinement of the Learning Strategies Scales. As in the study of Motivation scales, several iterations of EFA and CFA were conducted to refine the Learning Strategies scales. Since many of the item MSAs of the Learning Strategies tended to be lower than .5, the removal of cross-loading items took precedent to avoid removing too many items. However, all MSAs that were lower than .4 were removed to ensure acceptable adequacy. Table 7 shows the final Learning Strategies factor loadings.

Table 7. Final Learning Strategies Scales Factor Loadings

\begin{tabular}{|l|c|c|c|c|}
\hline \multirow{2}{*}{} & \multicolumn{5}{|c|}{ Component } \\
\hline & $\mathbf{1}$ & $\mathbf{2}$ & $\mathbf{3}$ & $\mathbf{4}$ \\
\hline LS43_ER & .804 & & & \\
\hline LS38_E & .801 & & & \\
\hline LS42_TS & .793 & & & \\
\hline LS10_SR & .751 & & & \\
\hline LS45_SR & .751 & & & \\
\hline LS50_E & .750 & & & \\
\hline LS17_ER & .719 & & & \\
\hline LS28_R & .711 & & & \\
\hline LS33_E & .699 & & & \\
\hline LS22_E & .692 & & & \\
\hline LS37_HS & .685 & & & \\
\hline LS46_TS & & .701 & & \\
\hline LS6_ER & & .686 & & \\
\hline LS29_ER & & .682 & & \\
\hline LS2_SR & & .667 & & \\
\hline LS49_TS & & .652 & & \\
\hline LS26_SR & & .595 & & \\
\hline LS24_SR & & & .782 & \\
\hline LS35_CT & & & .749 & \\
\hline LS16_CT & & & .702 & \\
\hline LS8_HS & & & .546 & \\
\hline LS32_O & & & & .762 \\
\hline LS41_R & & & & .739 \\
\hline LS36_E & & & & .728 \\
\hline LS18_O & & & & .655 \\
\hline
\end{tabular}


The survey items were examined in an attempt to re-label the factors. Factor 1 consisted of items from many of the original scales: four items from Elaboration, two items from Effort Regulation, two items from Metacognitive SelfRegulation, one item from Time and Study Environment, one item from Help Seeking, and one item from Rehearsal. Although most items were from the original Effort Regulation scale, it was decided to re-label this factor as General Strategies for Learning, as used in the study conducted by Dunn, Lo, Mulvenon, and Sutcliffe [3]. The wordings in items loaded on Factor 2 included "trying to understand," "I either give up or only study the easy parts," "distracted by other things," and "I quit." All these items were closely related to one's ability to regular one's effort to stay on task, so this factor was re-labeled as Effort Regulation. Factor 3 consisted of items that include "experiment on the computer," "play around with different software functions," "practice the computer functions," and "test it using software." Two of these items were from the original Critical Thinking scale. Since questioning the software's functionality and experimenting with software to find its capability can be interpreted as using critical thinking skills, this factor was re-labeled as Critical Thinking. The items loaded on Factor 4 included wording such as "write brief summaries," "make lists," and "go over class notes." Two of these were from the original Organization scale, and it was decided to re-label this factor as Organization.

A path model was created based on this EFA. The model fit characteristics of the CFA included a CFI of .815, a GFI of .86, and a RMSEA of .076. Although these did not reach the desired CFI of $>.92$, GFI of $>.92$ for a good fit, it was determined that they were at the acceptable level. Convergent validity was exhibited: all path coefficients were statistically significant and all factor loadings were higher than .5 with exception of item 26 (.491), which were very close to the desired .5. In addition, all four scales exhibited discriminant validity: the root average variance explained was larger than correlations in the same row and column in the table. Table 8 shows the comparison of root average variance and inter-scale correlations for the final Learning Strategies Scales.

Table 8. Comparison of Root Average Variance Explained and Inter-Scale Correlation for the Final Learning Strategies Scales

\begin{tabular}{|c|c|c|c|}
\hline Critical Thinking & $\begin{array}{c}\text { General Strategies for } \\
\text { Learning }\end{array}$ & Organization & Effort Regulation \\
\hline 0.690 & & & \\
\hline 0.425 & 0.725 & & \\
\hline 0.606 & 0.009 & 0.704 & \\
\hline-0.159 & 0.191 & -0.530 & 0.597 \\
\hline
\end{tabular}

Reliability. Cronbach's Alpha was calculated for each of the scales. All Cronbach's Alpha met the desired .7; $1^{\text {st }}$ $(.920), 2^{\text {nd }}(.782), 3 \mathrm{~d}(.817)$, and $4^{\text {th }}(.792)$. No deletion of any item in each of these sub-scales would raise the scale Alpha.

\section{CONCLUSIONS}

It was very clear that the survey items in both the Motivation section and in the Learning Strategies section did not confirm to the original MSLQ scales. Both Motivation and Learning Strategies path models exhibited very poor model fit and no validity. After several iterations of refinements, the Motivation section contains 24 items with 3 scales: Expectancy, Test Anxiety, and Task Value. Of the three scales, only Test Anxiety retained the original items. One item in Task Value scale was from the original Intrinsic scale. Items from Intrinsic, Extrinsic, Task Value, Control Beliefs, and Self-Efficacy were loaded to the same factor: Factor 1. This result was similar to that of the research conducted by Hilpert et al. [5]. Their latent structure, for the Motivation section, consisted of expectancy (self-efficacy and control of learning beliefs) and value (intrinsic motivation and task value). This final Motivation section with Expectancy, Test Anxiety, and Task Value scales exhibited convergent validity and discriminant validity. The scales also had good internal consistency of reliability with Cronbach's coefficient alphas exceeded the desired .7.

The initial EFA did not produce clear loadings for the Learning Strategies scales, and the model failed both convergent validity and discriminant validity tests. After several iterations of refinements, the final latent structure consisted of only four factors: Effort Regulation, Organization, Critical Thinking, and General Strategies for Learning (GSL). The GSL scale was used to represent the wide range of original scales from which the items loaded in this new scale. Based on the EFA, items from six of the original nine scales loaded on this factor. These new scales also exhibited convergent 
validity and discriminant validity, although the fit statistics were only at an acceptable range. However, all four scales exhibited good internal consistency of reliability with Cronbach's coefficient alphas exceeded .7.

The original The Motivated Strategies for Learning Questionnaire (MSLQ) provides a solid foundation for research into context-based disciplines. Development and validation of an adapted instrument to measure students' motivation and learning strategies within computer-based learning environments presented in this study contributes to the legacy of the MSLQ develop by Pintrich et al. [9]. The innovative instrument used in this study can be used to improve teaching and learning objectives within the information systems field of study. The adapted MSLQ survey may contribute to the development of information systems course delivery and academic performance assessment. Future research that clarifies the relationships between motivational beliefs and learning strategies could improve students'

learning management systems that advance academic performance. Finally, researchers must continue to explore the complexities of student academic experiences and learning backgrounds in computer-based learning environments, and subsequently expand the theoretical framework.

\section{REFERENCES}

1. Cook, D., Thompson, W., \& Thomas, K. (2011). The motivated strategies for learning questionnaire: Score validity among medicine residents. Medical Education, 45, 1230-1240.

2. Cronbach, L. J., \& Shavelson, R. J. (2004). My current thoughts on coefficient alpha and successor procedures. Educational and Psychological Measurement, 64(3), 391-418.

3. Dunn, K. E., Lo, W.-J., Mulvenon, S. W., \& Sutcliffe, R. (2012). Revisiting the motivated strategies for learning questionnaire: A theoretical and statistical reevaluation of the metacognitive self-regulation and effort regulation sub-scales. Educational and Psychological Measurement, 72, 312-331.

4. Hair, J. F., Black, W. C., Babin, B. J., Anderson, R. E., \& Tatham, R. (2006). Multivariate Data Analysis: Pearson Education. Upper Saddle River, New Jersey.

5. Hilpert, J., Stemien, J., van der Hoeven Kraft, K., \& Husman, J. (2013). Evidence for the latent factor structure of the MSLQ: A new conceptualization of an established questionnaire. SAGE Open, 1-10.

6. Kane, M. (2001). Current concerns in validity theory. Journal of Educational Measurement 38: 319342.

7. Pintrich, P. \& DeGroot, E. (1990). Motivational and self-regulated learning components of classroom academic performance. Journal of Educational Psychology, 82, 33-40.

8. Pintrich, P., Smith, D., Garcia, T., \& McKeachie, W. (1991). A Manual for the Use of the Motivated Strategies for Learning Questionnaire. Ann Arbor, MI: University of Michigan.

9. Pintrich, P., Smith, D., Garcia, T., \& McKeachie, W. (1993). Reliability and predictive validity of the motivated strategies for learning questionnaire (MSLQ). Educational and Psychological Measurement, 53, 801-813.

10. Rotgans, J. \& Schmdt, H. (2009). Examination of the context-specific nature of self-regulated learning. Educational Studies, 35(3), 239-253. 